\title{
Contribution of Neural Elements to Thoracic Stability
}

\author{
Fatih Ersay DENIZ1, Phillip M. REYES², Bruno C.R. LAZARO², Felix C. DOMINGUEZ², Anna G.U.S. NEWCOMB², \\ Volker K.H. SONNTAG ${ }^{3}$, Neil R. CRAWFORD ${ }^{2}$ \\ ${ }^{1}$ Gaziosmanpasa University, Faculty of Medicine, Department of Neurosurgery, Tokat, Turkey \\ ${ }^{2}$ St. Joseph's Hospital and Medical Center, Barrow Neurological Institute, Spinal Biomechanics Laboratory, Phoenix, Arizona, USA \\ ${ }^{3}$ St. Joseph's Hospital and Medical Center, Barrow Neurological Institute, Division of Neurological Surgery, Phoenix, Arizona, USA
}

\section{ABSTRACT}

AIM: Studies of spinal biomechanics typically do not focus on the contributions to range of motion (ROM) of the primary components of the spinal canal, dura, arachnoid, pia, spinal cord, nerve roots, ligaments, and vessels. We sought to determine the stability offered by these soft tissues in vitro.

MATERIAL and METHODS: Human cadaveric segments were tested intact, after osteoligamentous destabilization, and after transection of T8-9 spinal canal components. Specimens were induced into flexion, extension, axial rotation, and lateral bending using non-constraining, non-destructive pure moment while tracking motion response stereophotogrammetrically. The range of motion (ROM) was compared in each condition after adjusting for soft tissue creep.

RESULTS: After spinal canal element transection, ROM increased in all directions (mean 4.7\%). This increase was most pronounced during lateral bending $(p=0.055)$. The cumulative ROM from all directions of loading showed a statistically significant mean increase of $3.3 \%(p=0.040)$.

CONCLUSION: Sectioning of canal elements was found to cause a measurable increase in ROM. Although nonviable tissues were tested, living tissues are also likely to contribute to spinal stability.

KEYWORDS: Destabilization, Range of motion, Spinal canal elements, Spine biomechanics

ABBREVIATIONS: LZ: Lax zone (zone of ligamentous/soft tissue laxity), PLL: Posterior longitudinal ligament, ROM: Range of motion, SZ: Stiff zone (zone of ligamentous/soft tissue stretching)

\section{INTRODUCTION}

$\mathrm{S}$ tudies of spinal biomechanics typically focus on the contributions to range of motion (ROM) of the ligaments, articulations, vertebral discs, and, to a limited degree, muscles. However, the primary contents of the spinal canal, dura, arachnoid, pia, spinal cord, nerve roots, ligaments, and vessels are usually not considered in biomechanical analyses. These structures also experience strain during movement and should therefore contribute to some limitations in ROM. The small contributions that these components make on ROM and the difficulty in isolating these tissues during a flexibility test are probable reasons for why their contributions have been ignored when ROM is studied. Published reports of biomechanical studies related to neural elements are mostly limited to monoaxial tensile tests and histological studies $(1,9,11)$. Although knowledge of the strengths and stiffness of individual elements is useful, the amount that the neural structures contribute to stability depends on the degree of interaction with adjacent connective tissues during different motions. The objective of this study was to evaluate the effects of thoracic spinal canal elements, especially the neural structures, on ROM. These findings should help us to better understand 
basic spinal biomechanical responses, which may be useful in interpreting biomechanical findings and developing more refined computer models of the spine.

\section{- MATERIAL and METHODS}

\section{Specimen Preparation}

Seven human cadaveric thoracic spines (T6-T11) with $3 \mathrm{~cm}$ of ribs attached were studied. IRB approval was not sought as it is not required for cadaveric studies using anonymized donor tissues. The mean age at the time of death was 47 years (range, 36-68 years). Unembalmed frozen specimens were obtained and thawed in a bath of normal saline at $30^{\circ} \mathrm{C}$. Residual muscle tissue was carefully stripped, preserving ligaments, discs, and joint capsules.

Visual and radiographic inspection and examination of history indicated that no specimen had pathological entities in the region being studied. Before testing, the rostral and caudal vertebrae were potted in metal fixtures using polymethyl methacrylate for the application of loads (15). The specimens were wrapped in saline-soaked gauze during testing to prevent dehydration.

\section{Biomechanical Testing}

A system of pulleys and cables were used with a standard servohydraulic test system (MTS Systems Corp., Eden Prairie, MN) to apply nondestructive, nonconstraining puremoment loads (4). Loads were applied around the appropriate anatomical axes to induce flexion, extension, right axial rotation, left axial rotation, right lateral bending, and left lateral bending (Figure 1). The specimens were preconditioned three times before data were collected in each loading mode. Preconditioning entailed application and holding a $7.5-\mathrm{Nm}$ load for 60 seconds. Each specimen was then allowed to recoil to a natural resting position and rest for 60 seconds. Loads were applied in $1.25-\mathrm{Nm}$ increments to a maximum of $7.5 \mathrm{Nm}$ during data collection, and each increment was held for 45 seconds.

Motion of each specimen was tracked using the Optotrak 3020 (Northern Digital, Inc., Waterloo, Ontario, Canada). This system allows for stereophotogrammetric measures of three -dimensional displacement of infrared-emitting markers which are rigidly attached in a noncollinear arrangement to three stainless steel surgical guidewires inserted into each vertebra. Custom software aligned the local Cartesian coordinate systems at T7-T8, T8-T9, and T9-T10 with the vertebral anatomy by using a digitizing probe and converting the marker coordinates on T7, T8, T9, and T10 to angles around the anatomical axis of each motion segment relative to its caudal vertebra $(5,7)$.

Each specimen was tested in the following conditions: 1) intact, 2) after osteoligamentous destabilization, and 3) after canal component sectioning. The specimens were kept wrapped in saline-soaked gauze to prevent dehydration during testing. If a specimen required a second day of testing, the specimen was stored in a cold room until testing resumed to mitigate degradation from prolonged exposure to room temperatures.

\section{Surgical Procedures}

The osteoligamentous destabilization procedure at T8-9 consisted of the following surgical procedures: a rightsided transpedicular discectomy, division of the anterior longitudinal ligament, transection of the anterior aspect of the disc with a scalpel from foramen to foramen, sectioning of the posterior portion of the disc with a curette to allow for preservation of the posterior longitudinal ligament (PLL), bilateral total facetectomy, and sectioning of the interspinous and supraspinous ligaments (Figure 2). Canal component sectioning consisted of perpendicular sharp transection of the spinal cord at the T8-9 level using a scalpel, scissors, and a curette. After all tests were conduced, complete dissection of each specimen was performed to verify that the components had been properly sectioned.

\section{Data and Statistical Analysis}

From the raw data, three parameters were generated from the quasistatic load-deformation data: angular ROM, lax zone (LZ, zone of ligamentous/soft tissue laxity), and stiff zone (SZ, zone of ligamentous/soft tissue stretching). The ROM can be divided into $L Z$ and $S Z$, which thus are components of ROM. The LZ represents the low- stiffness portion of the typically biphasic load-deformation curve, while SZ represents the highstiffness portion (6). The LZ is similar to Panjabi's neutral zone,

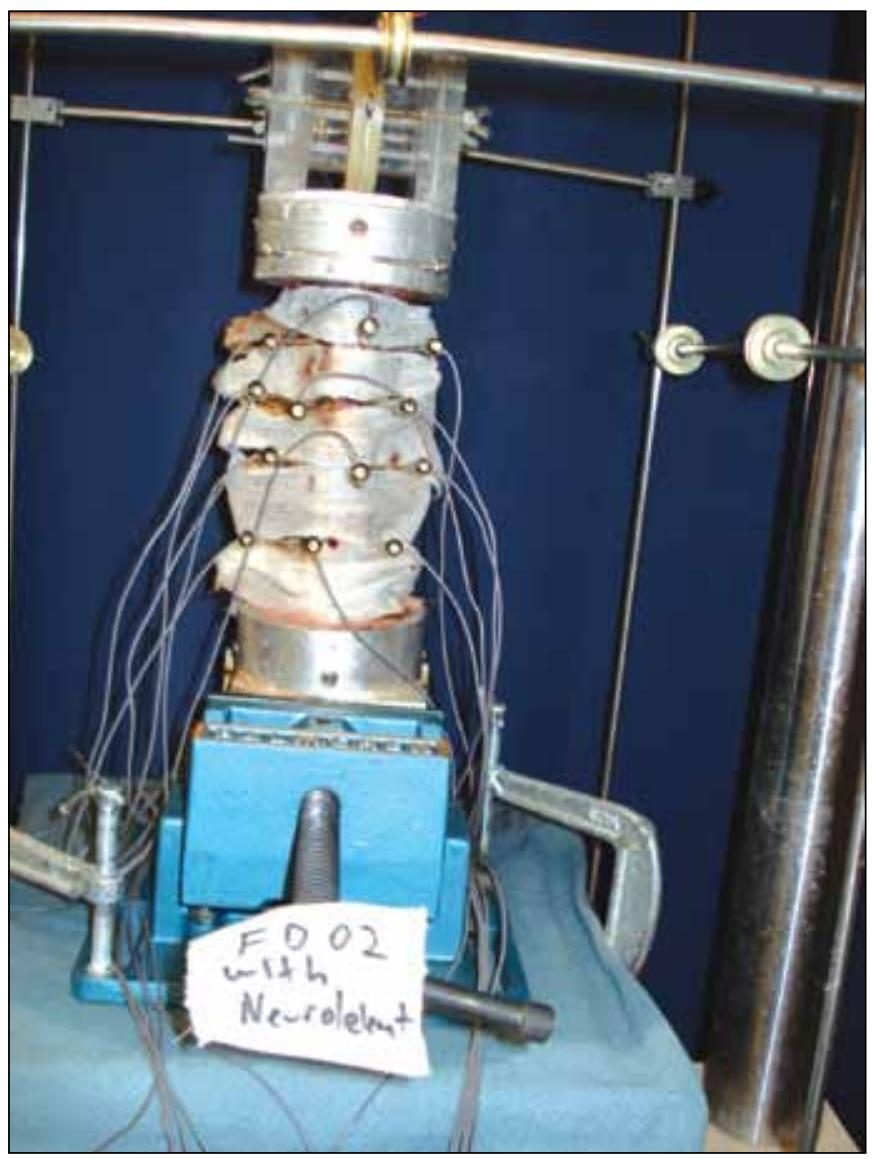

Figure 1: A representative specimen during biomechanics testing, optical markers for tracking motion are visible. 
which is defined as the zone in which there is only frictional joint resistance (12). LZ is more more reproducible and refers to the zone in which there is minimal ligamentous resistance (6). The location at which LZ crossed to SZ was calculated by extrapolating the load-deformation slope at data points

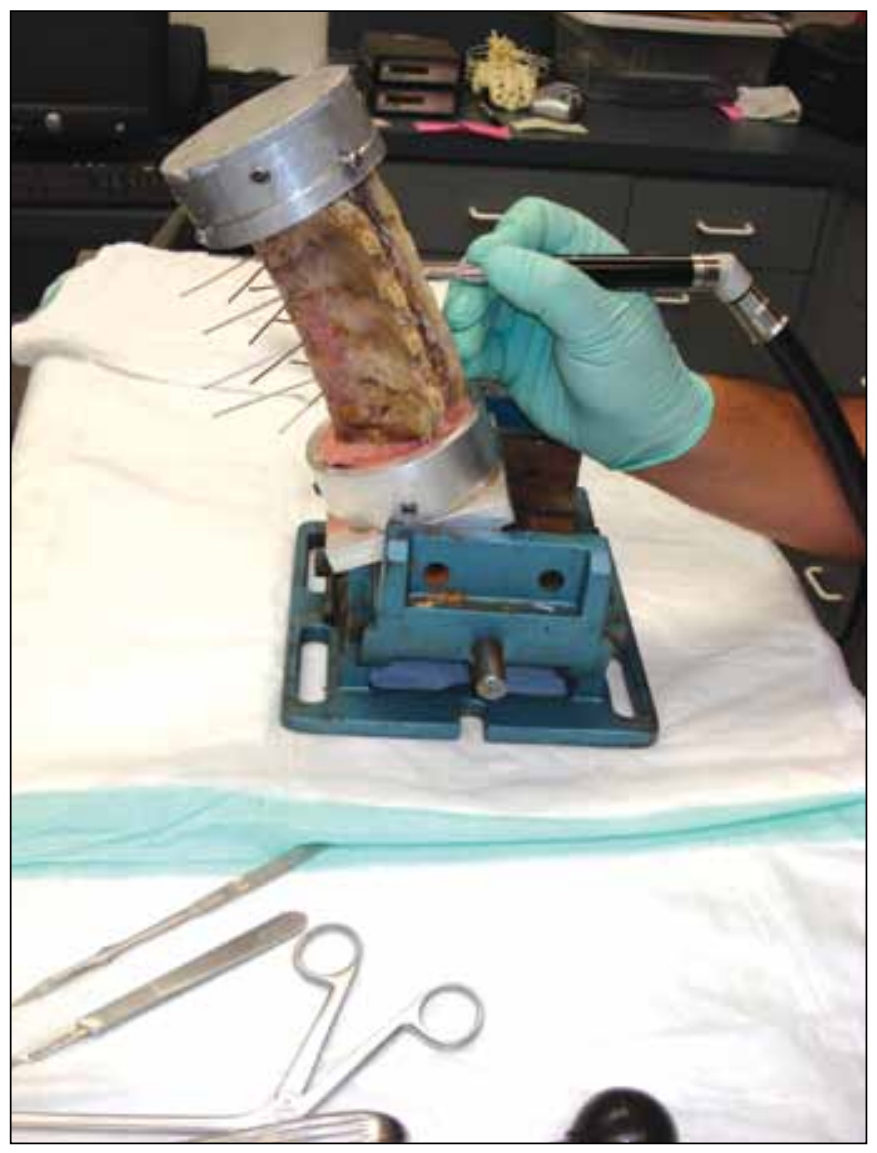

Figure 2: Photograph of surgical procedure. corresponding to $3.75 \mathrm{Nm}, 5.0 \mathrm{Nm}, 6.25 \mathrm{Nm}$, and $7.5 \mathrm{Nm}$ to zero load using the method of least squares in Microsoft Excel. Larger values of LZ, SZ, or ROM indicate greater instability. The angular ROM during flexion, extension, axial rotation, and lateral bending was the maximum angular displacement under the maximum applied load. ROM (including LZ and SZ) was expressed in degrees. Before statistical analysis, ROM values (including LZ and SZ) were normalized to account for the natural degradation of specimens from repeated testing. Normalization was done by adjusting the ROM at the index level (T8-9) based on variations in ROM at the adjacent levels. The mean ROM increase at T7-8 and T9-10 in each direction of loading was used to create a scaling factor $F$ :

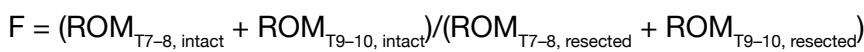

This scaling factor was multiplied by the ROM (or LZ) at the corresponding index-level. Thus, the increase in motion related to natural degradation was controlled so that the normalized increases in motion at the index level represented only the effect of the transections. Repeated- measures analysis of variance followed by Holm-Sidak tests were used to determine whether motion parameters differed significantly following transection. Probability values less than 0.05 were considered significant.

\section{RESULTS}

After the neural elements were transected, the mean normalized ROM at the index level increased by $1.6 \%$ during flexion-extension $(p=0.310), 8.1 \%$ during lateral bending $(p=0.055)$, and $4.4 \%$ during axial rotation $(p=0.171)$ (Figure 3 , Table I). Analysis of cumulative normalized ROM (the combined normalized ROM from all 4 directions of loading) showed a mean increase in ROM of $1.08 \pm 0.96$ degrees, corresponding to $3.3 \%(p=0.040)$. There was an increase in the mean normalized $L Z$ after neural element transection by $0.2 \%$ during flexion-extension $(p=0.887), 8.1 \%$ during lateral

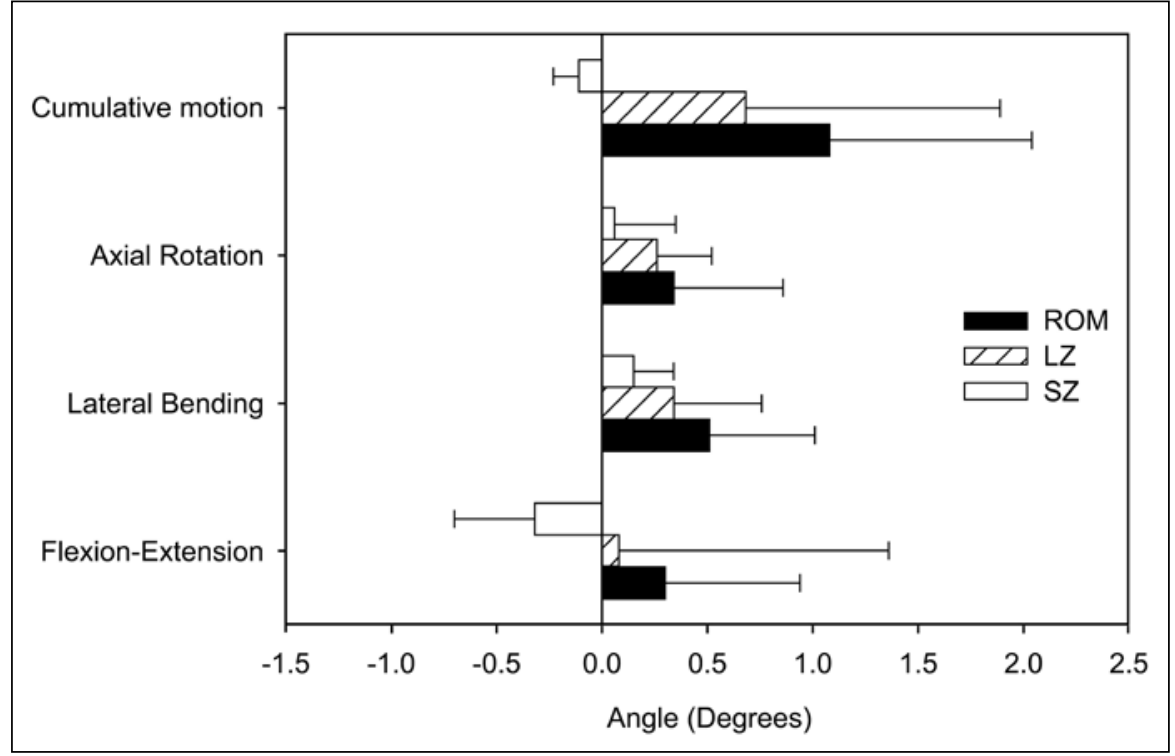

Figure 3: Mean changes in normalized ROM, LZ, and SZ (degrees) at the index level (T8-9) in different directions of loading, after transection of the neural elements. Cumulative motion refers to all directions of loading combined (Flexion-Extension + Lateral Bending + Axial Rotation). A negative value indicates a decrease. Error bars show standard deviation. (Used with permission from Barrow Neurological Institute, Phoenix, Arizona, USA). 
Table I: Mean Percent Increases in Normalized ROM, LZ, and SZ at the Index Level (T8-9) in Different Directions of Loading, Following Transaction of the Neural Elements*

\begin{tabular}{lccc}
\hline Direction of motion & ROM & LZ & SZ \\
\hline Flexion-Extension & $+1.6 \%(p=0.310)$ & $+0.2 \%(p=0.887)$ & $-6.0 \%(p=0.090)$ \\
\hline Lateral Bending & $+8.1 \%(p=0.055)$ & $+8.1 \%(p=0.108)$ & $+6.9 \%(p=0.111)$ \\
\hline Axial Rotation & $+4.4 \%(p=0.171)$ & $+5.3 \%(p=0.056)$ & $+2.1 \%(p=0.655)$ \\
\hline Cumulative & $+3.3 \%(p=0.040)$ & $+3.0 \%(p=0.229)$ & $-1.1 \%(p=0.060)$ \\
\hline
\end{tabular}

LZ: Lax zone, ROM: Range of motion, SZ: stiff zone.

${ }^{*} P$ values indicate outcome of repeated measures analysis of variance (RM-ANOVA)/Holm-Sidak tests relative to intact. Cumulative motion refers to all directions of loading combined (Flexion-Extension + Lateral Bending + Axial Rotation). A negative value indicates a decrease.

bending $(p=0.108)$, and $5.3 \%$ during axial rotation $(p=0.056)$. The mean cumulative normalized $L Z$ increased by $0.68 \pm 1.21$ degrees, corresponding to $3.0 \%(p=0.229)$. After, neural element transection, the mean normalized $\mathrm{SZ}$ decreased by $6.0 \%$ during flexion-extension $(\mathrm{p}=0.090)$, increased by $6.9 \%$ during lateral bending $(\mathrm{p}=0.111)$, and increased by $2.1 \%$ during axial rotation $(p=0.655)$. The mean cumulative normalized SZ decreased overall by $0.11 \pm 0.12$ degrees, which corresponds to $1.1 \%(p=0.060)$.

\section{DISCUSSION}

Although in biomechanical analyses the spinal canal elements are not traditionally considered to be contributors in ROM, the spinal canal elements are part of a dynamic mechanical system that should be recognized (16). This study is the first to examine the contribution of spinal canal components to the stability of the thoracic spine.

The central region of the anterior dural sac adheres to the deep layer of the PLL at its midline. It has been suggested that these areas where the dural sac adheres to the PLL are due to anterior dural ligaments (Hoffmann's ligaments). These ligaments have been observed to change direction along the spine, from caudocranial (dura to PLL) at upper thoracic levels to transverse at the level of T8-9 to craniocaudal at lower thoracic and lumbar levels, often with multiple ligaments being present at a single level. The limitation to movement that these ligaments provide may be an important factor in protecting the spinal cord and spinal nerves from trauma during movement, particularly during extension of the vertebral column (19).

The spinal dura is composed of collagen and elastic fibers and is primarily a protective membrane for the spinal cord $(10,14,18,20)$. Collagen fibers of the dura are oriented mainly longitudinally and have higher tissue resistance, stronger tensile strength, and greater stiffness in the longitudinal direction than in the transverse direction $(13,14,20)$. The elastin content of spinal dura has been determined in cadavers (11), and was found to be approximately 2 times higher in the dorsal aspect compared to the ventral aspect (13.8\% vs. $7.1 \%$ of the total dry weight, respectively). In comparison, the same investigators report an elastin content of $7.3 \%$ of the total dry weight of the PLL. The elastic modulus of the dura mater has been reported to be $5 \mathrm{MPa}$, compared to
$0.26 \mathrm{MPa}$ for the spinal cord itself, $35.2 \mathrm{MPa}$ for the anterior longitudinal ligament, 35.7 MPa for the PLL, and $35.7 \mathrm{MPa}$ for the dural attachments, including the Hoffmann's ligaments (9). Therefore, and because the position of the spinal cord is posterior to the axis of rotation, it would be expected that the spinal cord contributes less to restricting motion during extension than during flexion. Such behavior was indeed observed.

After the thoracic spinal canal components were sectioned, ROM was affected in all directions of movement; however, the increases were small. These small increases are not surprising considering the well-known main restrictors of motion (i.e., ligaments, discs, and articulations). However, knowing that the neural elements contribute slightly to ROM and determining the extent of this contribution is important for academic work and research.

This study has some limitations. Primarily, the tissues studies were not living, and therefore probably contribute less to stability than they would in-vivo because of deflation. In the same way that bending a deflated balloon is easier than bending an inflated balloon, the living spinal cord should have greater resistance to bending than cadaveric tissue. In a living body, muscles and pressure from the intact tissues within the torso would also contribute to ROM differently than in a cadaveric specimen $(2,3,8,17,20)$.

\section{- CONCLUSION}

This study demonstrates that the deformation capacity of the spinal canal contents in an in vitro model has a slight effect on ROM during nondestructive flexibility testing. This small effect was most pronounced during lateral bending. These data may be useful in creating a more complete model of the biomechanical response of the spine to external loads.

\section{REFERENCES}

1. Akaishi F: Biomechanical properties of the anterior and posterior longitudinal ligament in the cervical spine. Nihon Ika Daigaku Zasshi 62: 360-368, 1995

2. Bilston LE: The effect of perfusion on the soft tissue mechanical properties: A computational model. Comput Methods Biomech Biomed Engin 5: 283-290, 2002 
3. Bilston LE, Thibault LE: The mechanical properties of the human cervical spinal cord in vitro. Ann Biomed Eng 24: 6774,1996

4. Crawford NR, Brantley AG, Dickman CA, Koeneman EJ: An apparatus for applying pure nonconstraining moments to spine in vitro. Spine (Phila Pa 1976) 20: 2097-2100, 1995

5. Crawford NR, Dickman CA: Construction of local vertebral coordinate systems using a digitizing probe. Technical note. Spine (Phila Pa 1976) 22: 559-563, 1997

6. Crawford NR, Peles JD, Dickman CA: The spinal lax zone and neutral zone: Measurement techniques and parameter comparisons. J Spinal Disord 11: 416-429, 1998

7. Crawford NR, Yamaguchi GT, Dickman CA: A new technique for determining 3-D joint angles: The tilt/twist method. Clin Biomech ( Bristol, Avon) 14: 153-165, 1999

8. Fiford RJ, Bilston LE: The mechanical properties of rat spinal cord in vitro. J Biomech 38: 1509-1515, 2005

9. Greaves CY, Gadala MS, Oxland TR: A three-dimensional finite element model of the cervical spine with spinal cord: An investigation of three injury mechanism. Ann Biomed Eng 36: 396- 405, 2008

10. Maikos JT, Elias RA, Shreiber DI: Mechanical properties of dura mater from the rat brain and spinal cord. J Neurotrauma 25: 38-51, 2008

11. Nakagawa $\mathrm{H}$, Mikawa $\mathrm{Y}$, Watanabe $\mathrm{R}$ : Elastin in the human posterior longitudinal ligament and spinal dura. A histologic and biochemical study. Spine (Phila Pa 1976) 19: 2164- 2169, 1994
12. Panjabi MM: The stabilizing system of the spine. Part II. Neutral zone and instability hypothesis. J Spinal Disord 5: 390-396; discussion 397, 1992

13. Patin DJ, Eckstein EC, Harum K, Pallares VS: Anatomic and biomechanical properties of human lumbar dura mater. Anesth Analg 76: 535-540, 1993

14. Runza M, Pietrabissa R, Mantero S, Albani A, Quaglini V, Contro R: Lumbar dura mater biomechanics: Experimental characterization and scanning electron microscopy observations. Anesth Analg 88: 1317-1321, 1999

15. Scholz M, Reyes PM, Schleicher P, Sawa AG, Baek S, Kandziora F, Marciano FF, Crawford NR: A new stand-alone cervical anterior interbody fusion device: Biomechanical comparison with established anterior cervical fixation devices. Spine (Phila Pa 1976) 34: 156-160, 2009

16. Tunituri AR: Elasticity of the spinal cord dura in the dog. $J$ Neurosurg 47: 391-396, 1977

17. van Dieen $\mathrm{JH}$, van der Veen $\mathrm{A}$, van Royen BJ, Kingma I: Fatigue failure in shear loading of porcine lumbar spine segments. Spine (Phila Pa 1976) 31: E494-498, 2006

18. Vandenabeele F, Creemers J, Lambrichts I: Ultrastructure of the human spinal arachnoid mater and dura mater. J Anat 189 (Pt 2): 417-430, 1996

19. Wadhwani S, Loughenbury $P$, Soames $R$ : The anterior dura (Hofmann) ligaments. Spine (Phila Pa 1976) 29: 623-627, 2004

20. Zarzur E: Mechanical properties of the human lumbar dura mater. Arq Neuropsiquiatr 54:455-469, 1996 